\title{
Mathematical modelling of parasitic infections : from data and parameter estimation to evolutionary implications
}

\author{
EdTed by MARÍA-GLORIA BASÁÑEZ \\ CO-ORdinating EDITOR L. H. CHAPPELL
}

\section{Preface}

As the discipline of parasitology has become increasingly quantitative, mathematical models have become widely accepted as useful tools with which to formalise our current understanding of the population biology of parasites, their transmission dynamics, and to explore the impact on such dynamics of control interventions. The field of mathematical modelling of parasitic infections has moved on a great deal from the pioneering work of Ross and Macdonald, followed by that of Tallis and Leyton, Bailey, Nåssell, Dietz, and Anderson and May to mention a few, yet relatively simple, single parasite and host models continue to offer profound qualitative insights and stimulate much needed research to test the hypotheses that are implicit or explicitly formulated in such models. In earlier stages of development are models to tackle the issue of multiple infections of a host, multiple hosts of a pathogen, or those merging principles of population dynamics and population genetics to investigate the consequences of control interventions both in epidemiological and evolutionary scales.

This special issue of Parasitology was conceived as a result of two sessions on modelling parasitic infections at the 11th International Congress of Parasitology (ICOPA XI), held in Glasgow during the summer of 2006, and which I had the pleasure to chair with Ian Hastings from the University of Liverpool. The sessions were very successful and with most speakers willing to contribute their papers, my co-editor Les Chappell and I felt brave enough to embark in the preparation of this volume. The selection of articles we now present contains papers from the ICOPA sessions and others that we commissioned subsequently. We start the volume as we intend to end it, with papers that take into account possible evolutionary implications of control interventions. Exactly 100 years ago Sir Ronald Ross initiated, in his report on Mauritius in 1908, the development of mathematical models for the study of malaria epidemiology, so we see it fit to begin this special issue with a section on models for malaria control. Thus, the article by Jacob Koella and Lamia Zaghloul examines the prospects of using evolutionary costs to try and enhance the efficacy of controlling this infection through the use of genetically modified mosquitoes, and concludes that even when combining evolutionary costs (decreased fitness of resistant mosquitoes) with epidemiological costs (decreased survival of infected mosquitoes), the fraction of resistant mosquitoes that has to be achieved to effect a meaningful impact is still very high. The paper by Alan Saul continues this theme by exploring models for mosquito stage transmission blocking vaccines and concludes that we may be closer to an effective transmission blocking vaccine than is widely believed. Yet, it is not obvious how decreased Plasmodium oocyst intensities in mosquitoes translate into given levels of vaccine efficacy. The final paper of this 'malaria models' section describes a comprehensive micro-simulation model by Tom Smith and co-workers, with the potential of providing a platform for testing, under different epidemiological scenarios, the likely impact of the control measures explored in the previous two papers alone or in combination with other interventions.

The contribution of Olivier Restif and coauthors challenges the commonly made assumption 
in models for multi-strain pathogen dynamics that cross immunity between strains is protective and symmetric, by modelling experimentally-derived data regarding the agents of whooping cough, Bordetella pertussis and B. parapertussis in mice. The next two papers link the themes of microparasitic and macroparasitic infections with Andrea Pugliese and Roberto Rosà discussing the effect of host population densities on tick-borne diseases and ectoparasitic tick abundance, and Andy Fenton proposing for the first time a deterministic model for the population dynamics of microparasite and macroparasite coinfection that is used to explore how introduction of a macroparasite into a host population already harbouring a microparasite infection alters the predictions of existing single-species models, and the conditions for stability and persistence.

The last section of this special issue focuses on models for helminth infections, with the paper by Sarah Perkins and collaborators using an empirically-derived social network structure to examine the relative roles of host mixing patterns and male mouse-biased transmission of Heligmosomoides polygyrus among rodent hosts. Christian Bottomley and co-authors fit a Bayesian hierarchical model to field-derived microfilarial data obtained during a control trial to estimate fertility rates of female Onchocerca volvulus after repeated ivermectin treatment, and Wilma Stolk and colleagues present a micro-simulation model for the transmission and control of Anopheles-transmitted Wuchereria bancrofti in African settings. Finally Penelope Lynch and co-workers contest the view that antiparasitic measures that kill adult worms will select for smaller, less fecund helminths, and argue that evolutionary outcomes will in fact depend on the precise nature of the relationship between the mortality rates affected by treatment, worm size and developmental status. The three last papers have potentially important repercussions for our understanding of rates of transmission stage production, reinfection, and spread of anthelmintic resistance following long-term chemotherapy.

Therefore, this collection of papers covers a wide range of systems, from vector-borne infections to directly-transmitted parasites, from models based on infection prevalence to those based on infection intensity, and from those using deterministic approaches to those based on stochastic simulations. Important issues include interactions between pathogen strains and micro- and macroparasitic infections, the role of network structure and the importance of evolutionary considerations, and the usefulness of mathematical models both as thinking tools for qualitative understanding or as guiding tools for decision support in major control programmes. We thank the continued support of the editorial team of Parasitology and hope that our readers will enjoy this volume as much as we have enjoyed preparing it.

MARÍA GLORIA BASÁÑEZ August 2008 